\title{
Open Services for IoT Cloud Applications in the Future Internet
}

\author{
*Martin Serrano ${ }^{1}$, Hoan Nguyen M. Quoc ${ }^{1}$, \\ Manfred Hauswirth ${ }^{1}$ \\ ${ }^{1}$ National University of Ireland - NUI Galway \\ Digital Enterprise Research Institute (DERI) \\ Galway, Ireland \\ \{firstname.lastname\}@deri.org
}

\author{
Wei Wang ${ }^{2}$, Payam Barnaghi ${ }^{2}$ \\ ${ }^{2}$ University of Surrey \\ Centre for Communication \\ Systems Research (CCSR) \\ Guilford, United Kingdom \\ \{wei.wang; p.barnaghi\}@surrey.ac.uk
}

\author{
Philippe Cousin ${ }^{3}$ \\ ${ }^{3}$ Easy Global Market \\ Nice, France
}

philippe.cousin@eglobalmark.com

\begin{abstract}
Internet of Things (IoT) is an emerging area that not only requires development of infrastructure but also deployment of new services capable of supporting multiple, scalable (cloud-based) and interoperable (multi-domain) applications. In the race of designing the IoT as part of the Future Internet architecture, academic and ICT's (Information and Communication Technology) industry communities have realized that a common IoT problem to be tackled is the interoperability of the information. In this paper we review recent trends and challenges on interoperability, and discuss how semantic technologies, open service frameworks and information models can support data interoperability in the design of the Future Internet, taking the IoT and Cloud Computing as reference examples of application domains.
\end{abstract}

Keywords- Intenet of Things; Open Services; Interoperability; Semantics; Future Internet, Cloud Services, Cloud Computing.

\section{INTRODUCTION}

Current Internet architecture as a design conception does not include free information exchange between data and service levels; this fact promotes a race between academic and industry for designing the Future Internet in which how to enable information interoperability is a key challenge [1][2][3]. As a negative result of this race, a fully coordinated course in terms of design and implementation has not occurred, due to many complex issues involving mainly deployment over particular diverse infrastructures. The new design requires transformation from an agnostic to a more service and network aware Future Internet.

Convergence towards Internet technologies for communications networks and services has been a clear trend in the ICT domain in the past few years. Challenges in the future communications systems mainly involve Internetbased systems demand, in terms of end user requirements, personalized provisioning, service-oriented performance, and service-awareness networking. To support these demands information interoperability and data model integration are crucial. Future Internet has been described in previous works [1][2]. In this paper the intention is to discuss the Future Internet in a service-oriented manner, coming through a revision about the role semantic web and their methods of exposing, annotating, sharing and connecting information (linked-data) can play to satisfy the afore mentioned challenges. In the Future Internet, services and networks follow a common design guideline; provide solutions in form of cause-open interoperable mechanisms.

Internet is suffering a radical shift from traditional networked-switched environment with heavy/complex protocols and signalling focused on applications-oriented perspective, towards a converged service-oriented architecture (SOA) space. Research initiatives addressing this SOA and cloud-based requirement argue that the future lies in layers of overlay networks that can meet various requirements whilst keeping a very simplistic, almost unmanaged, IP for the underlying Internet, GENI NSFfunded initiative to rebuild the Internet [4]. Others argue that the importance of wireless access networks requires a more fundamental redesign of the core Internet Protocols themselves [5][6]. RINA approach [7] is based on the fundamental principle that networking is an inter-process communication (IPC) and it recourses the IPC services over different scopes to enable networking operations-services. Whilst this debate continues, it is a consensus that information interoperability among heterogeneous data models is necessary.

We follow the idea that service agnostic design is not anymore a way to achieve interactive solutions in terms of service composition and information sharing capabilities for heterogeneous infrastructures. Instead a more holistic and long-term view is required, in which networking issues are addressed in a manner that various protocols delivering services meet rapidly the changing needs of users.

Figure 1 depicts a service-aware Future Internet holistic view where information is shared at different levels and its implementation relies on the Linked-Data layer or inference plane [8], where the exchange of information facilitates 
knowledge-driven composed services by enabling interoperable management support and generation and exchange of information. So the move towards converged IP-based communications networks increasingly provides solutions to a number of significant technical issues by using more standard information exchange, promoting information interoperability and allowing the networks to be managed effectively. More importantly, offering open opportunities for a knowledge-based, service-oriented support can have a fundamental impact on future communications networks and services.

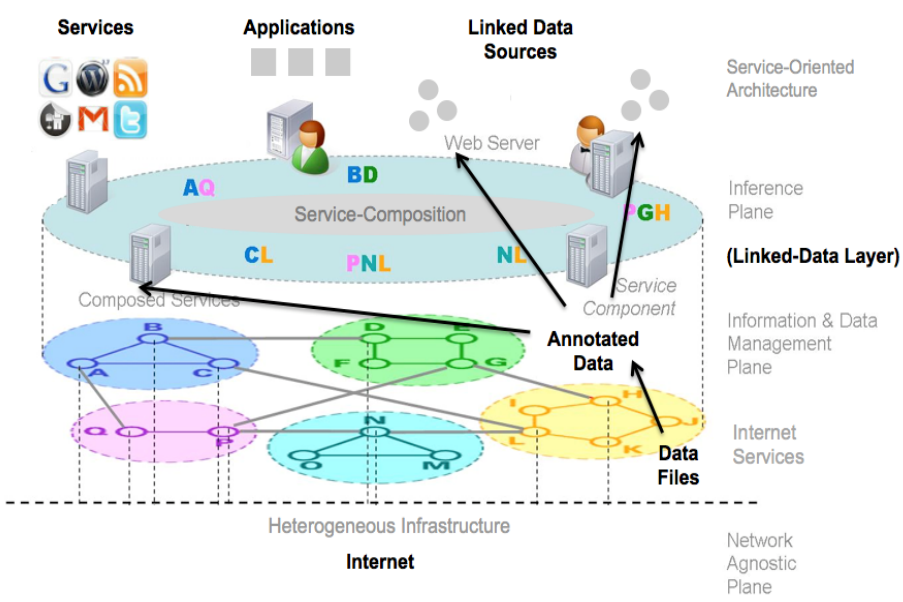

Figure 1. Service Openness by meaning of Linked-Data Interoperability.

This paper focuses on information interoperability and Linked Data for controlling communication systems in the future networks and services in cloud environments. The extensible, reusable, common and manageable information Linked-Data layer is critical for this deployment and follows cloud principles. The novelty aspect of this approach relies on the fact that high-level infrastructure representations do not use resources when they are not required to support or deploy services. The optimization of resources using this approach relies on classifying and identifying what resources need to be used, by semantic descriptions in a knowledge-based fashion. Thus the service composition is dynamically executed and cloud-based services deployed by result of knowledge-based analysis.

Organization of this paper is as follows: Section II presents the summary of challenges for an interoperable Future Internet based on Linked Data, IoT and Cloud, where information exchange occurs to support management of cloud services and network infrastructure. Section III introduces Linked Data approach to facilitate information interoperability with the semantic descriptions. Section IV introduces two exemplar open service middleware for cloud-based systems interoperability. Section V presents the summary and outlook.

\section{CHALlENGES FOR IOT IN THE FUTURE INTERNET}

Taking a broad view of state of the art, current development of data link interactions and converging communications, many of the problems present in current Internet about data and information management are generated by interoperability problems, we have identified three of these persistent problems:

1. Users are offered relatively small numbers of Internet services, which they can not personalize to meet their evolving needs; communities of users can not tailor services to help create, improve and sustain their social interactions;

2. The Internet services that are offered are typically technology-driven and static, designed to maximize usage of capabilities of underlying network technologies and not to satisfy user requirements per se, and thus cannot be readily adapted to their changing operational context;

3. Internet operators cannot configure their networks to operate effectively in the face of changing service usage patterns and rapid networking technology deployment; networks can only be optimized, on an individual basis, to meet specific low-level objectives, often resulting in sub-optimal operation in comparison to the more important business and service user objectives.

As the move towards convergence of communications networks and services, a more extended service-oriented architecture design, gains momentum worldwide facilitated mainly by pervasive deployment of Internet protocol suites, VoIP is a clear example of this. The academic research community is increasingly focussing on how to evolve networking technologies to enable the "Future Internet". In this sense by addressing evolution of networking technologies in isolation is not enough; instead, it is necessary to take a holistic view of the evolution of communications services, their societal drivers and the requirements they will place on the heterogeneous communications infrastructure over which they are delivered [9][10].

By addressing information interoperability challenge issues, Internet systems can reflect changing individual and societal preferences in network and services and can be effectively managed to ensure delivery of critical services in a services-aware design view with general infrastructure challenges. IoT information interoperability challenges addressed at the data and service levels cannot be addressed alone without considering the overall interoperability framework because IoT needs to be seen as:

a) high-dimensional, with the co-existence of many systems (devices, sensors, equipment, etc.) in the environment that need to communicate and exchange information; 
b) highly-heterogeneous, where vast number of systems are designed for different purposes and targeted at diverse application domains, making it extremely difficult (if not impossible) to reach out for global agreements and widely accepted specification;

c) dynamic and non-linear, where new Things (that were not even considered at start) are entering (and leaving) the environment all the time. Therefore, new unforeseen formats and protocols need to be supported to communicate and share data in the IoT;

d) hard to describe/model, existence of many data formats and modelling principles, that often cannot be interrelated with one another.

The framework for sustainable interoperability in the IoT needs therefore and at least to address other aspects coordinated with the approach promoted in this paper and in particular ensuring technical interoperability of the enabling protocols, or aspect of dynamic (cognitive) interoperability methods allowing things to dynamically interoperate within the heterogeneous world. IERC [11] is working with the IoT research community to develop an overall interoperability framework, which can cope with all the challenges, discussed in this article as important elements to consider.

\section{LINKED DATA: AdVANTAGES AND BENEFITS}

A current activity in many research and development communities is the composition of data models for enabling information management control. It focuses on using semantic models (Ontology models) by means of annotated data to enable extensible, reusable, common and manageable data plane. Previously referenced as inference plane [8], the knowledge-based approach plane uses semantic technologies for supporting interoperability and the required extensibility in handling end-user contents for pervasive applications.

The concept of the Linked Data originates from the need to interlinking individual data items and information objects to support semantic query and inferences on the data coming from the physical and virtual objects. The Linked Data, represented using formal knowledge representation formalisms, (i.e., the collection of semantic technologies such as RDF [12] and OWL [13][14]), provides potential for information reuse and interoperability among heterogeneous sources. Different from the traditional ways of publishing information where datasets are simply made available on the Web, information published for the Linked Data is structured and connected to each other using logical constructs in widely used ontologies, such as FOAF [15] and SKOS [16], to form the Web of data. The principles of publishing the Linked Data encourages reusing of existing information rather creating new one. Applications and human users can exploit the existing knowledge base by simply providing links to the data in it. DBpedia ${ }^{1}$ is one of the most notable examples of the Linked Data, which extracts structured information from the Wikipedia. It enables not only sophisticated queries over the amazing amount of crowd-sourced information but also new ways of browsing and navigation through the semantic links.

The IoT manifests high degree of heterogeneity in many ways from the different types of things in the real world to different models for representing those things. The resulting interoperability problem makes identifying, discovering and searching things on the global scale on the Internet a challenging task. In the past few years, researchers have proposed to use the semantic and service oriented technologies to address the interoperability issue. Many of the research efforts have been focussing on developing semantic models for annotating the things in the IoT domain. One of the most notable works in this line is the development of the W3C's Semantic Sensor Network (SSN) ontology [17]. The SSN ontology aims to model the sensors (and sensor networks), which are one category of the things. Later, the work in [18] has tried to provide a holistic modelling of the IoT domain concepts (e.g., entity of interests, physical and indoor locations, unit of measurement and so on); in particular, to hide the heterogeneity and complexity of the underlying devices (e.g., sensors), the modelling for services is proposed. The idea behind this is that a service exposes the functionalities that can be provided by the devices and these functionalities can be semantically described using service models [19]. The combination of the semantic and service oriented techniques can support both interoperability and scalability for the IoT.

Having semantic models and ontologies alone is not sufficient to achieve interoperability in the IoT. Ontologies developed by different parties are not guaranteed to be compatible with each other; in many cases, the research on ontology matching can be used to align the different ontologies; however, accuracy of the matching is frequently not satisfying and significant amount of human effort is still needed. Ontology reuse has been seen as an effective way to alleviate this problem; however, we suggest that bringing the Linked Data principles to the IoT is more substantial: reusing of the concepts at schema level is important, while reusing the instances or linking to the instances in existing knowledge bases is even more important. By linking to existing knowledge rather than creating repetitive one helps a Web of interlinked IoT data to facilitate navigation, discovery and more importantly, interoperability among different sources. We have seen many recent research works that make use of the Linked Data in IoT research [20][21]; however, to realise higher level of interoperability, the IoT community needs to make data publically available and link it to the existing knowledge to create a better connected linked-data plane.

${ }^{1} \mathrm{http}: / /$ dbpedia.org/ 


\section{OPEN SERVICES AND SYSTEMS INTEROPERABILITY}

Semantic interoperability is based on the composition of data models for enabling information management control. It focuses in the semantic enrichment task of the management information described in both enterprise and networking data models with semantic Linked Data to provide an extensible, reusable, common and manageable inference plane between the Internet-based designs and cloud-based data processing systems.

A) Super Stream Collider (SSC) is a platform, which provides a web-based interface and tools for building sophisticated mashups combining semantically annotated linked stream and Linked Data sources into easy-to-use resources for applications. The system includes drag\&drop construction tools along with a visual SPARQL [22]/ CQELS [23]. editor and visualization tools for novice users while supporting full access and control for expert users at the same time (Figure 2). Tied in with this development platform is a cloud deployment architecture which enables the user to deploy the generated mashups into a cloud, thus supporting both the design and deployment of stream-based web applications in a very simple and intuitive way.

A deployment of SSC is available online ${ }^{2}$, which provides a user- friendly interface called SSC visual editor. This is a light-weight Web-based workflow editor for composing mashup data through drag\&drop. Using the SSC visual editor, we aim at providing a programmable Web environment suitable not only for expert users but also for non-expert programmers.

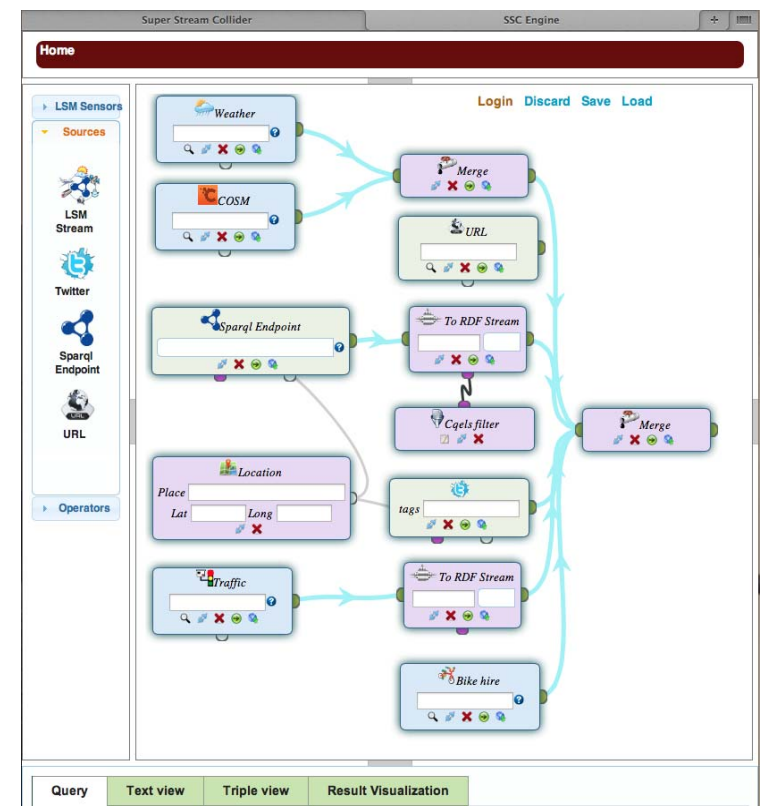

Figure 2. Super Stream Collider User Graphic Interface

Figure 3 provides an overview screenshot of SSC visualization. The main components of the platform are the visual editor (1), data sources and operators (2) and the debugging component (3). Each data source and operator is visualized as a block in the editor area Visually, a mashup workflow is a combination of connected operators and data sources. It is incrementally designed by dragging the icons representing for corresponding operators in (2) and then dropping to the editor area (1).

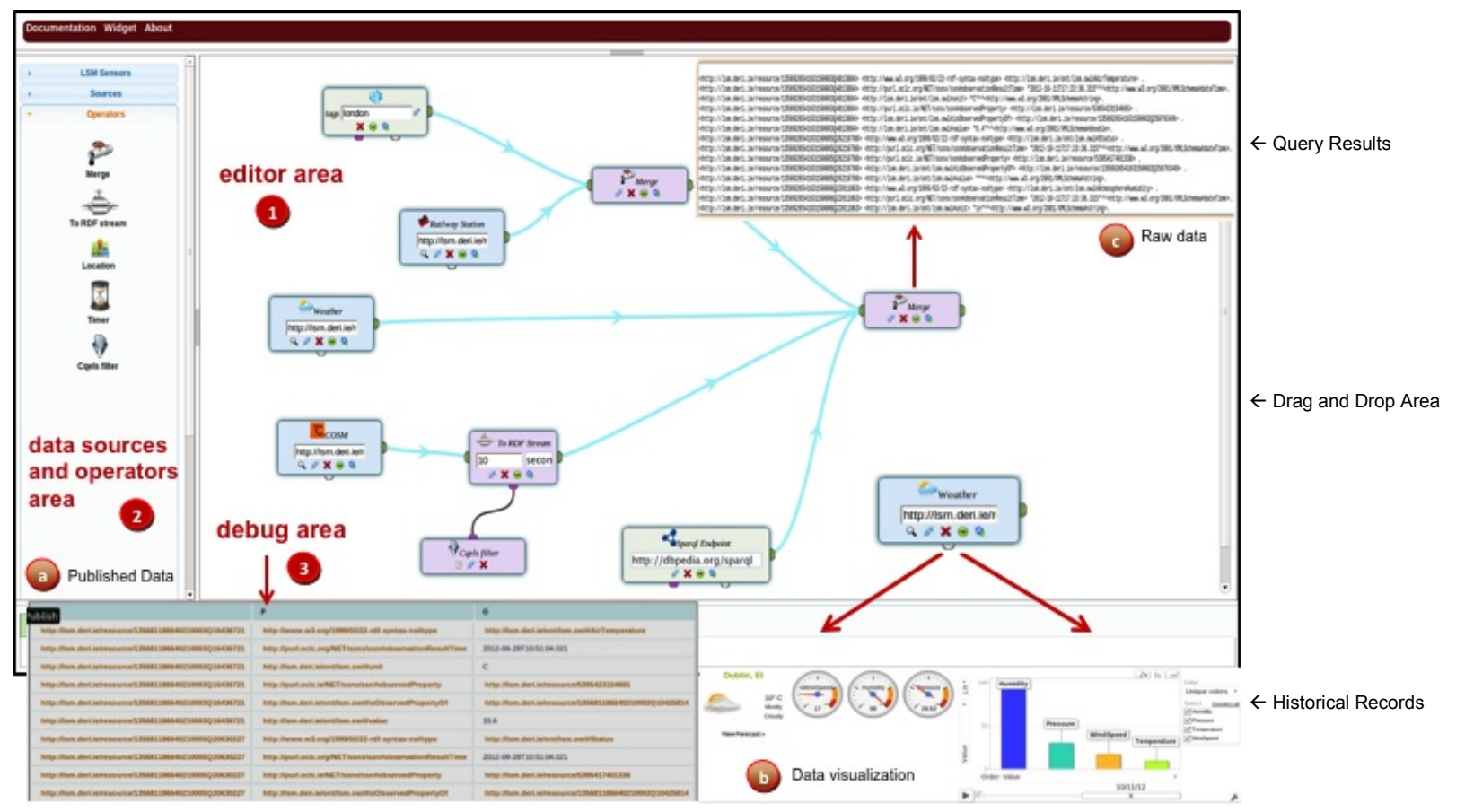

Fig. 3. Super Stream Collider drag \& drop interface

${ }^{2}$ http://superstreamcollider.org/ showing editor, data sources and operators and debug areas. 
The flows of data from the sources to the final output are defined by wiring the blocks with configured parameters. The live visualizations of operator outputs are shown in (3). The output of the workflow is a live mashup data stream which can be published, visualized and queried. Currently SSC supports several types of live data sources, such as LSM [24] sensors (over 100,000 sensor around the world including weather, train schedule, traffic status, etc.), twitter streams, DBPedia and Sindice ${ }^{3}$ data sources, among others, which can be discovered by the SSC discovery component. This context-aware discovery service uses relevant text, location, sensor data sources that the user has typed and chosen as inputs to form the queries to such systems to find useful data items to recommend to the user (a) in Figure 3 shows a temperature sensor as an example. SSC's debugging component supports the user by showing the results of each of his/her actions (c) and its historical records (b).

B) Sense2Web provides a semantic annotation framework to describe sensor devices and services. The semantically annotated sensor data is published as linked RDF data and then made available to other applications via SPARQL endpoints. The annotations describe the sensor device/resource according to a semantic model that described in [18]. Sense2Web also provides a mash-up application using the published data and open data resource on the Web to demonstrate reasoning and interpretation of linked sensor data and resource descriptions.
Figure 4 shows the user interface for publishing a new sensor description in Sense2Web. The systems uses Jena API [25] to query DBpedia ${ }^{4}$ and GeoNames ${ }^{5}$ resources to obtain location, type and other attributes that are used for tagging and description of the sensor devices/services.

In the picture are shown suggestions that are retrieved from DBpedia for a sample query, "'Guildford"'. The submitted variables are stored in XML format and Extensible Stylesheet Language Transformations (XSLT) is used to transform the submitted data to RDF form. This makes generation of the RDF data flexible and less dependent to the current model. This allows Sense2Web annotation framework to link the sensor descriptions to other resources on the Web of Data and the constructed description can be changed by altering the XML form and XSL templates (as long as the required data and attributes are provided).

\section{CONCLUSIONS}

In the Future Internet there are high demands for information interoperability and Linked Data to enable automated service composition. As the requirements for automated composition of large number of open services are defined by diverse and heterogeneous systems, it is challenging to make complex system management operations in the absence of high degree of interoperability. The Linked Data emerges as an ideal solution to resolve part of the complex information interoperability issues in the Future Internet of networks and cloud.

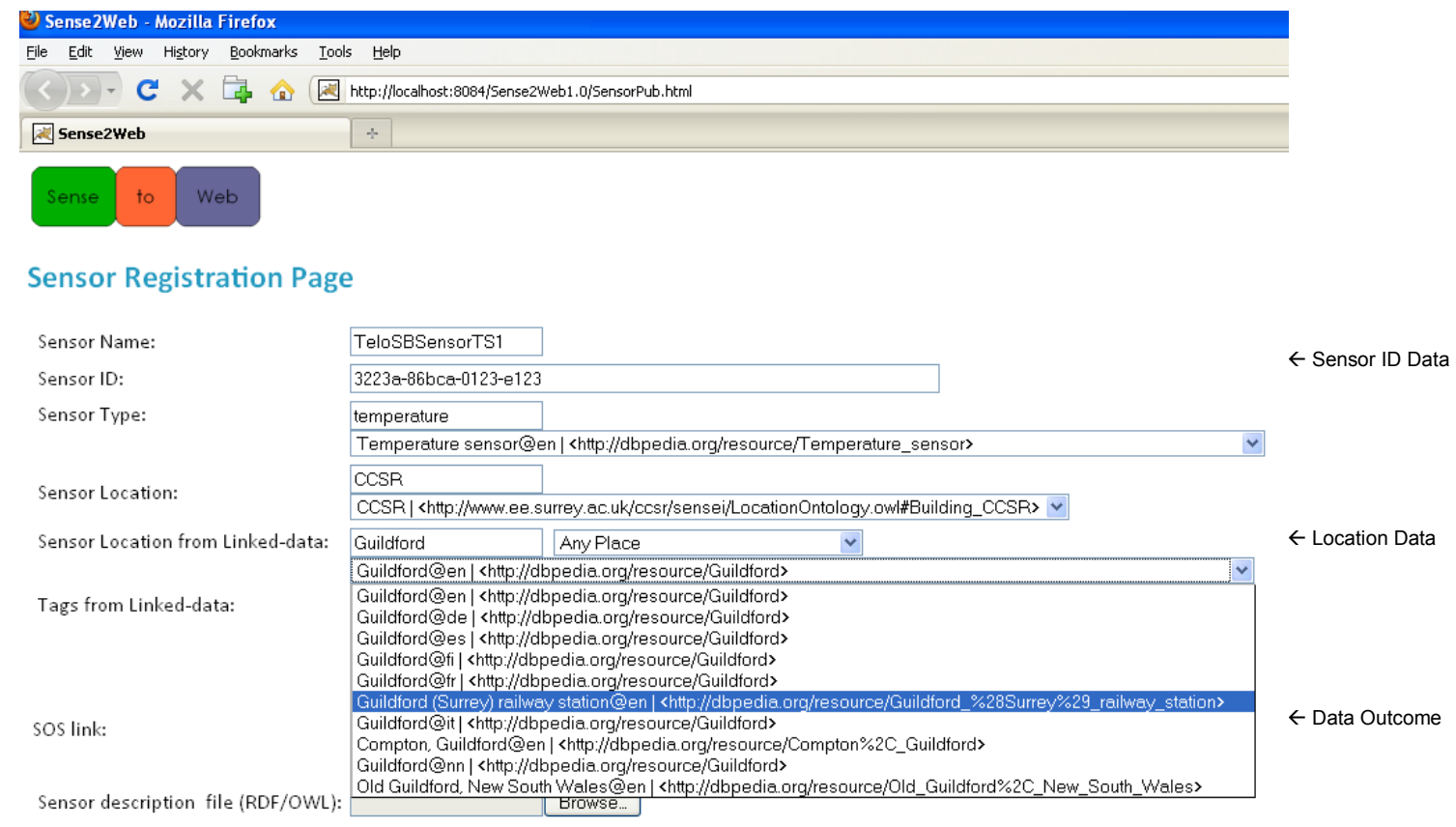

\footnotetext{
${ }^{3} \mathrm{http}: / /$ sindice.com/

${ }^{4} \mathrm{http}: / /$ dbpedia.org/

${ }^{5} \mathrm{http}: / /$ www.geonames.org/
}

Figure 4. Sensor data Publication interface with concept suggestions from DBpedia for "Guilford" sample keyword. 
This paper makes references to formal foundations for the development of interoperability of information by using Linked Data techniques, such semantic annotation, best practices and standards in Web science to exchange information. Linked data supporting information interoperability is a crucial requirement in Cloud-based services in the Future Internet. Their implications for network infrastructure and cloud-based services are still an open issue for research.

Interoperability and information exchange must be validated via direct industrial investment, and roll out on real integrated test beds to trial Future Internet infrastructures (potentially overlay networks or new inference planes) for cloud-based services.

We have studied and demonstrated how formal representation of Linked Data for the service information facilitates information interoperability in service composition and management processes. Remaining research challenges regarding information model extensibility and information dissemination in cloud systems exist and would be conducted to conclude implementations and experiments composing the discussed cloud-based Linked Data services.

\section{ACKNOWLEDGMENT}

This work has been carried out in the Framework of the European Research Cluster on Internet of Things (IERC-AC4) Cluster Activity Group 4 - Service openness and inter-operability issues / semantic interoperability, and has been co-funded by the European Commission FP7 framework program under the following EU ICT projects: OpenIoT Project (Open source blueprint for large scale self-organizing cloud environments for IoT applications) ${ }^{6}$, IoT.est Project (Internet of Things Environment for Service Creation and Testing) ${ }^{7}$ and ProbeIoT project (Pursuing ROadmaps and BEnchmarks for the Internet of Things) ${ }^{8}$.

\section{REFERENCES}

[1] D. Clark, et al., "NewArch: Future Generation Internet Architecture", NewArch Final Technical Report, http://www.isi.edu/newarch/

[2] M. Blumenthal, D. Clark, "Rethinking the design of the Internet: the end to end arguments vs. the brave new world", ACM Transactions on Internet Technology, Vol. 1, No. 1, Aug. 2001

[3] A. Feldmann, "Internet clean-slate design: what and why?," ACM SIGCOM Computer Communication Review, Vol. 37, Issue 3, 2007

[4] NSF-funded initiative to rebuild the Internet, http://www.geni.net/

[5] Clean Slate program, Stanford University, http://cleanslate.stanford.edu

[6] Architecture Design Project for New Generation Network, http://akari-project.nict.go.jp/eng/index2.htm

\footnotetext{
${ }^{6} \mathrm{http}: / /$ www.openiot.eu/

${ }^{7}$ http://ict-iotest.eu/

${ }^{8} \mathrm{http}: / /$ www.probe-it.eu/
}

[7] John Day. Patterns in Network Architecture: A Return to Fundamentals. Published by Prentice Hall (ISBN 0132252422 ), December 2007.

[8] M. Serrano, J. Strassner and M. ÓFoghlú, “A Formal Approach for the Inference Plane Supporting Integrated Management Tasks in the Future Internet" 1 st IFIP/IEEE ManFI International Workshop, In conjunction with 11th IFIP/IEEE IM2009, 1-5 June 2009, at Long Island, NY, USA.

[9] Martín Serrano, Steven Davy, Martin Johnsson, Willie Donnelly, Alex Galis, "Designs of Federated Management in Future Internet Architectures". Chapter book in Future Internet Assembly Book, Future Internet: Achievements and Promising Technology, by Springer, May 2011

[10] Future Internet Assembly, http://www.futureinternet.ie

[11] IERC-Internet of things European Research Cluster www.internet-of-things-research.eu . Activity Chain 4 on Service openness and inter-operability issues/semantic interoperability

[12] W3C Website.

http://www.w3.org/TR/PR-rdf-syntax/

[13] Horridge, M., Knublauch, H., Rector, A., Stevens, R., Wroe, C., "A Practical Guide to Building OWL Ontologies using the Protégé-OWL Plugin and CO-ODE Tools Edition 1.0" Manchester University, August. 2004.

[14] OWL Ontology Web Language. http://www.w3.org/2004/OWL

[15] FOAF. http://www.foaf-project.org

[16] SKOS. http://www.w3.org/2001/sw/wiki/SKOS

[17] W3C Semantic Sensor Network Incubator Group Report http://www.w3.org/2005/Incubator/ssn/XGR-ssn-20110628/

[18] S. De, P. Barnaghi, M. Bauer, S. Meissner, "Service modelling for the Internet of Things", in Proceedings of the Conference on Computer Science and Information Systems (FedCSIS), pp.949-955, Sept. 2011.

[19] Wang W, De S, Toenjes R, Reetz E, Moessner K, "A Comprehensive Ontology for Knowledge Representation in the Internet of Things", International Workshop on Knowledge Acquisition and Management in the Internet of Things in conjunction with IEE IUCC, 25-27 June, 2012.

[20] P. Barnaghi, M. Presser, K. Moessner, "Publishing Linked Sensor Data", in Proceedings of the 3rd International Workshop on Semantic Sensor Networks (SSN), November 2010.

[21] W. Wang, S. De, G. Cassar, K. Moessner, "Knowledge Representation in the Internet of Things: Semantic Modelling and its Applications", special issue on Knowledge Acquisition and Management in the Internet of Things, Automatika, 2012.

[22] SPARQL. http://www.w3.org/TR/rdf-sparql-query/

[23] D.Le-Phuoc,M.Dao-Tran, J.X.Parreira, and M.Hauswirth. A native and adaptive approach for unified processing of linked streams and linked data. In ISWC'11, October 2011.

[24] Danh Le-Phuoc, Hoan Nguyen Mau Quoc, Josiane Xavier Parreira, and Manfred Hauswirth, The Linked Sensor Middleware: Connecting the real world and the Semantic Web, at 9th Semantic Web Challenge co-located with 10th International Semantic Web Conference - ISWC 2011, October 23-27, 2011 Bonn, Germany.

[25] Jena, 2008, jena, A Semantic Web Framework for Java, http://jena.sourceforge.net/. 